\title{
Light scattering from a rough metal surface: theory and experiment
}

\author{
Roberto Li Voti, ${ }^{1, *}$ Grigore L. Leahu, ${ }^{1}$ Simone Gaetani, ${ }^{1}$ Concita Sibilia, ${ }^{1}$ Vittorio Violante, ${ }^{2}$ Emanuele Castagna, ${ }^{2}$ and \\ Mario Bertolotti ${ }^{1}$ \\ ${ }^{1}$ Dipartimento di Energetica, Sapienza Università di Roma, Via A. Scarpa 16-00161 Roma, Italy \\ ${ }^{2}$ ENEA C.R. Frascati, via Enrico Fermi 45-00044 Frascati, Italy \\ *Corresponding author: roberto.livoti@uniroma1.it
}

Received March 3, 2009; accepted April 27, 2009;

posted June 2, 2009 (Doc. ID 108183); published July 15, 2009

\begin{abstract}
There is still great interest in the determination of microtopographic properties of rough metallic surfaces from light scattering measurements. According to Beckmann-Kirchhoff theory a clear relationship is established between the in-plane angular scattered light intensity and the statistical properties of the surface. We discuss one way to invert this relationship, and we introduce a new iterative procedure to retrieve the height autocorrelation function even for a very rough metallic surface (rms surface roughness of the same order of the optical wavelength). The procedure is eventually applied to the experimental data of a known metallic surface for validation. () 2009 Optical Society of America

OCIS codes: $240.0240,240.3695,290.5880,120.5820$.
\end{abstract}

\section{INTRODUCTION}

The characterization of rough surfaces by light scattering has remained an active research field. Although the use of the real space imaging techniques, such as atomic-force microscopy (AFM) and scanning-tunneling microscopy, allow the surface morphology to be probed directly, indirect methods based on the light scattering from the sample surface still keep relevant advantages such as the contactless methodology, large sampling size, and the capability to detect nanotopographic surface features, heterogeneous nanostructures, nano-objects, etc.

The original idea to correlate surface roughness with light scattering came out from the early work of Lord Rayleigh [1,2]. However a systematic determined effort was made to solve the scattering problem for random surfaces only after World War II with the development of radar systems [3-5]. At that time myriads of approximated models [6-9] came out, but all used the BeckmannKirchhoff theory, where the surface scattering is explained as merely a diffraction phenomenon resulting from random phase variations induced on the reflected wavefront by (micro)topographic surface features, as described in the monograph by Beckmann and Spizzichino [10]. Nowadays the surface scatter phenomenon continues to be an important issue in diverse areas of science (optics, acoustics, geophysics, and terrestrial or extraterrestrial remote sensing), [11] and the limit of the validity of Beckmann-Kirchhoff theory is still under discussion, especially in optics [12-14].

The great interest in the Beckmann-Kirchhoff theory arises mainly because it establishes a clear relationship between the statistical surface properties (such as the rms roughness $\sigma$ and the autocorrelation function $C$ of the topographic surface) and the angular light scattering distribution. In the particular case of small roughness (with respect to the optical wavelength), many authors have shown that this relationship reduces to a simple Fourier transform that can be easily inverted with the standard methods known from signal processing [15-19].

In this paper we focus the study on the case of moderate to high surface roughness, for which the relationship is strictly nonlinear and consequently not invertible. We discuss the full theory and some crucial theoretical aspects of the inverse problem, discussing a new iterative procedure for retrieving the statistical parameters of the surface. First the procedure is tested on some numerical simulations, and eventually it is applied to some experiments of light scattering measured on a known rough metallic sample. The statistical properties obtained from the light scattering technique are eventually discussed and compared with the ones obtained with standard methods (stylus and AFM).

\section{THEORY}

We summarize in this paragraph the main theoretical results of the Beckmann-Kirchhoff theory, for better readability of the paper and comprehension of the critical points [10].

The rough metallic surface is represented by the function $\zeta(x, y)$, which has mean level $z=0$. Important related statistical quantities are the rms roughness $\sigma=\sqrt{\left\langle\zeta^{2}\right\rangle}$ (the brackets indicate averaging over the whole surface of interest) and the autocorrelation function $C=\left\langle\zeta_{1} \zeta_{2}\right\rangle$ ( $\zeta_{1}$ and $\zeta_{2}$ represent the heights of points $B_{1}$ and $B_{2}$ at a relative distance $\tau$, as shown in Fig. 1). $C$ is usually normalized to its maximum value $C_{\max }=\left\langle\zeta_{1} \zeta_{1}\right\rangle=\left\langle\zeta^{2}\right\rangle=\sigma^{2}$, occurring when $\tau=0\left(\mathrm{~B}_{1} \equiv \mathrm{B}_{2}\right)$.

A plane wave launched in the direction of the wavevector $\vec{k}_{i}$ and incident on the surface with the angle $\theta_{i}$ (with respect to normal) generates the disturbance at point $B$ (see Fig. 1): 


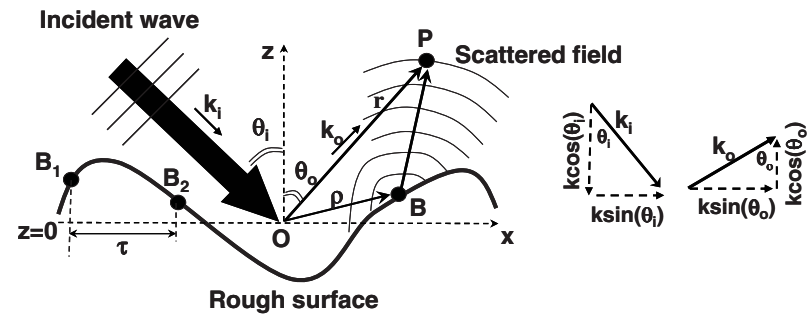

Fig. 1. Surface reflection is closely related to the microscopic surface profile and to the direction of the incident wave.

$$
V_{B}=A \exp \left\lfloor i \vec{k}_{i} \cdot \vec{\rho}\right\rfloor,
$$

where $\vec{\rho}$ is the vector $\mathbf{O B}$ and $A$ is a constant. Point B becomes the source of a secondary spherical wave that generates the disturbance at observation point $\mathrm{P}$ at distance $r$ chosen, without loss of generality, in the plane of incidence:

$$
\begin{aligned}
\psi & =\frac{\exp [i k|\vec{r}-\vec{\rho}|]}{|\vec{r}-\vec{\rho}|} \cong \frac{\exp \left[i k \sqrt{r^{2}+\rho^{2}-2 \vec{r} \cdot \vec{\rho}}\right]}{r} \\
& \cong \frac{\exp \left[i k r-i \vec{k}_{o} \cdot \vec{\rho}\right]}{r}
\end{aligned}
$$

where the wavevector $\vec{k}_{o}$ points in the direction of $\mathrm{P}$. It is worth noting that Eq. (2) is valid in the Fraunhofer zone of diffraction. The total scattered field at point $\mathrm{P}$ may be obtained according to the Helmholtz equation by integrating over the whole surface $S$ as follows [10]:

$$
\begin{aligned}
V_{P} & =\frac{1}{4 \pi} \int_{S}\left(V_{B, \text { tot }} \frac{\partial \psi}{\partial n}-\psi \frac{\partial V_{B, \text { tot }}}{\partial n}\right) \mathrm{d} S \\
& =F \cdot D \int_{S} \exp \left[i\left(\vec{k}_{i}-\vec{k}_{o}\right) \cdot \vec{\rho}\right] \mathrm{d} S,
\end{aligned}
$$

where $V_{B \text {,tot }}$ is the total disturbance at B (the sum of the incident and reflected terms), $F=\left[1+\cos \left(\theta_{i}\right.\right.$ $\left.\left.+\theta_{o}\right)\right] /\left[\cos \theta_{i}\left(\cos \theta_{i}+\cos \theta_{o}\right)\right]$ is an angular function obtained by many authors under the assumption of a perfectly conductive surface [10], $\theta_{i}$ and $\theta_{o}$ are the incident and the scattering angles, and $D$ is an unessential constant function that is dependent on the intensity and phase of the incident plane wave. The intensity may be obtained by averaging Eq. (3) as follows:

$$
I_{P}=\left\langle V_{P} V_{P}^{*}\right\rangle=F^{2} \int_{S^{\prime}} \int_{S}\left\langle\exp \left[i\left(\vec{k}_{i}-\vec{k}_{o}\right) \cdot\left(\vec{\rho}-\vec{\rho}^{\prime}\right)\right]\right\rangle \mathrm{d} S \mathrm{~d} S^{\prime},
$$

where the constant $|D|^{2}$ has been neglected because unessential (in the experiments the light scattering intensity is always normalized). Under the assumption of a normally distributed surface with a Gaussian statistic, Eq. (4) eventually becomes (see Eq. (44) of [10], p. 87)

$$
\begin{aligned}
I\left(\theta_{o}\right)= & 2 \pi F^{2} \int_{0}^{\infty} J_{o}\left[\frac{2 \pi}{\lambda}\left(\sin \theta_{i}-\sin \theta_{o}\right) \tau\right] \\
& \cdot \chi\left(\theta_{i}, \theta_{o}, \frac{\sigma}{\lambda}, C(\tau)\right) \cdot \tau \cdot \mathrm{d} \tau,
\end{aligned}
$$

where $\tau$ is the distance between two points $B_{1}$ and $B_{2}$ on the surface, $C(\tau)$ is the normalized autocorrelation function, and $\chi\left(\theta_{i}, \theta_{o}, \sigma / \lambda, C(\tau)\right)=\exp \left[-(2 \pi / \lambda)^{2} \sigma^{2}\left(\cos \theta_{i}\right.\right.$ $\left.\left.+\cos \theta_{o}\right)^{2}(1-C(\tau))\right]$. This equation establishes a clear relationship between the statistical properties of the surface and the angular scattered intensity. Just for example we discuss two simple cases for the normalized autocorrelation function: (a) the exponential case $C_{a}(\tau)=\exp (-\tau / \Lambda)$, where $\Lambda$ is the autocorrelation length (i.e., the distance at which $C$ decreases to $e^{-1}$ ), and (b) the linear case $C_{b}(\tau)$ $=1-\tau / \Lambda$ for which the scattered intensity $I_{b}$ may be expressed in the closed form [20]

$$
I_{b}\left(\theta_{o}\right)=\frac{2 \pi F^{2} \mu^{2}}{\left(\cos \theta_{i}+\cos \theta_{o}\right)^{4}}\left(\frac{\lambda}{2 \pi}\right)^{2} \frac{1}{\left[1+\frac{\left(\sin \theta_{i}-\sin \theta_{o}\right)^{2}}{\left(\cos \theta_{i}+\cos \theta_{o}\right)^{4}} \mu^{2}\right]^{3 / 2}},
$$

where $\mu=\lambda \Lambda / 2 \pi \sigma^{2}$. We performed the numerical simulations for both case (a) [by using $C_{a}$ in Eq. (5)], and case (b) [directly by using Eq. (6)] [21]. In Fig. 2 the normalized scattered intensity is shown also for different roughnesses $(\sigma=200,300 \mathrm{~nm})$, while $\lambda=633 \mathrm{~nm}, \Lambda=10 \mu \mathrm{m}$ have been kept constant and $\theta_{i}=0$. Note that the angular intensity profile becomes narrow as the roughness decreases as expected. Note also that there are no substantial differences between the exponential case (a) and the linear case (b), since $C_{a}(\tau)$ and $C_{b}(\tau)$ have similar initial behaviors. This makes Eq. (6) an excellent approximation for the light scattering from a metallic surface with an exponential autocorrelation function. Obviously Eq. (5) is more general and should be used for any other autocorrelation function $C(\tau)$; in fact we discuss in the next paragraph how Eq. (5) can be inverted in order to retrieve the unknown autocorrelation function.

It is worth noting that Eqs. (3)-(6) have been obtained in the case of perfectly conducting surface. In the case of finite conductivity of the metal, the surface partially re-

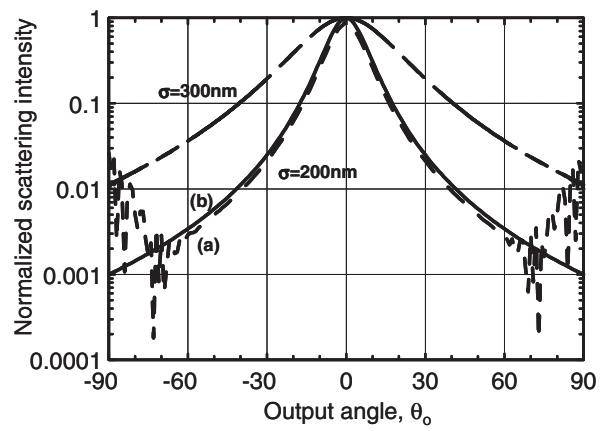

Fig. 2. Normalized angular scattered intensity versus scattering angle (degrees) for $\lambda=633 \mathrm{~nm}$ and autocorrelation length $\Lambda=10 \mu \mathrm{m}$. Curve (a) $\sigma=200 \mathrm{~nm}$ and $C_{a}(\tau)=\exp (-\tau / \Lambda)$; curve (b) $\sigma=200 \mathrm{~nm}$ and $C_{b}(\tau)=1-\tau / \Lambda$; dashed curve $\sigma=300 \mathrm{~nm}$ (the linear and the exponential case merge). 
flects the incident light, and the general problem becomes not trivial. However for many metals the reflectance is rather large and almost independent of the angle of incidence. In these cases Eqs. (3)-(6) are still valid in the present form, if one includes the average reflectance in the quantity $D$. This new quantity finally disappears in Eqs. (4)-(6) because of the standard normalization procedure.

\section{INVERSE PROBLEM}

In the previous paragraph we summarized the approach by Beckmann and Spizzichino for modeling the single scattering from random rough surfaces. Equation (5) evidences how the scattered intensity strongly depends on some particular quantities: the angle of incidence $\theta_{i}$, the angle of observation $\theta_{o}$, the optical wavelength $\lambda$, the rms roughness $\sigma$, and the roughness autocorrelation function $C(\tau)$. Note that the expression in Eq. (5) is not dissimilar from a 2D Fourier transform of the characteristic function $\chi$ from the domain $\tau$ to the spatial frequency domain $p$ $=\left(\sin \theta_{i}-\sin \theta_{o}\right) / \lambda$. As a simple check of validity we consider the particular case of a plane flat metallic surface for which $\sigma=0$. In this case the characteristic function is $\chi=1$ for any $C(\tau)$, and the scattered intensity indeed becomes the Dirac $\delta$ function (i.e., exactly the 2D Fourier transform of $\chi=1$ ) that is nonzero only for $\theta_{o}=\theta_{i}$, according to the well-known Snell law for specular reflection. But in the general case of a rough metallic surface the $\chi$ function also depends on $\theta_{o}$, making Eq. (5) slightly different from a rigorous 2D Fourier transform and making theoretically impossible the reconstruction of the $\chi$ function with an inverse Fourier transform procedure. Nevertheless, many authors [15-19] notice that for samples with a moderate surface roughness the scattered intensity is expected mainly in the quasi-specular direction for which $\theta_{o} \approx \theta_{i}$, where the $\chi$ function can be approximated as follows:

$$
\chi \asymp \exp \left[-\left(\frac{4 \pi \sigma \cos \theta_{i}}{\lambda}\right)^{2}(1-C(\tau))\right] .
$$

The result of such crucial approximation makes the integral in Eq. (5) formally equivalent to a 2D Fourier transform and invertible by means of the inverse Fourier (IF) transform as follows:

$$
\chi_{\mathrm{IF}}(\tau)=2 \pi \int_{0}^{\infty} J_{o}(2 \pi p \tau) \cdot \frac{I(p)}{F^{2}} \cdot p \cdot \mathrm{d} p,
$$

where $p=\left(\sin \theta_{o}-\sin \theta_{i}\right) / \lambda$ and $J_{o}$ is the Bessel function of the first kind. Combining Eqs. (7) and (8), the autocorrelation function $C_{\mathrm{IF}}$ is eventually retrieved as follows:

$$
C_{\mathrm{IF}}(\tau)=1-\left(\frac{\lambda}{4 \pi \sigma \cos \theta_{i}}\right)^{2} \ln \left(\frac{\chi_{\mathrm{IF}}(0)}{\chi_{\mathrm{IF}}(\tau)}\right)
$$

In practice a number of problems may limit the quality of the reconstruction.

(a) The integral in Eq. (8) is truncated when the maximum observation angle is reached at $\theta_{o}=\pi / 2$, corresponding to the maximum spatial frequency $p_{\max }=\left(1-\sin \theta_{i}\right) / \lambda$.
The limited bandwidth generally rounds the original profile $C(\tau)$ and decreases the spatial resolution of the reconstruction. The result of a numerical simulation is shown in Fig. 3(a), where the autocorrelation function $C=1-\tau / \Lambda$ with $\Lambda=10 \mu \mathrm{m}$ is reconstructed by Eq. (9). In the inset it is clear that the reconstruction $C_{\mathrm{IF}}$ (symbols) is initially rounded with respect to the original linear profile $\mathrm{C}$ (solid line).

(b) Since the scattered intensity is sampled with an angular step $\Delta \theta_{o}$, the reconstructed $C_{\mathrm{IF}}$ may be affected by some replica of $C(\tau)$, with a periodicity of the order of $\Delta \tau=\lambda / \Delta \theta_{o}$ (aliasing). In the numerical simulation we set $\Delta \theta_{o}=1^{\circ}$ so that the small replica could be visible only at a far distance of about $\tau \asymp 36 \mu \mathrm{m}$.

(c) For moderate roughness $\chi(\tau)$ tends to zero much faster than $C(\tau)$, making the reconstruction of $C_{\mathrm{IF}}$ by Eq. (9) more critical as $\sigma / \lambda$ increases. In Fig. 3(b) the $\chi$ function is shown for both $\sigma=200 \mathrm{~nm}$ (solid line) and $300 \mathrm{~nm}$ (dashed line). $\chi$ drops very quickly to zero, especially for $\sigma=300 \mathrm{~nm}$, as expected from Eq. (7), and the reconstructions of $\chi_{\mathrm{IF}}$ (symbols) fail down to the value $10^{-4}$, limiting the reconstruction interval till the distance $\tau_{\max }$ (i.e., for $\tau_{\max }=2 \mu \mathrm{m}$, inverted triangles, and for $\tau_{\max }=4 \mu \mathrm{m}$, dots). Correspondingly, the reconstructions of $C_{\mathrm{IF}}$ [symbols in Fig. 3(a)] clearly fail beyond $\tau_{\max }$.

In Fig. 4 the normalized scattering intensity spectra $I\left(\theta_{0}\right)$ are shown for the numerical examples reported in Figs. 3 (solid curve for $\sigma=200 \mathrm{~nm}$ and dashed curve for $\sigma$ $=300 \mathrm{~nm}$ ). To check the validity of the procedure the scat-
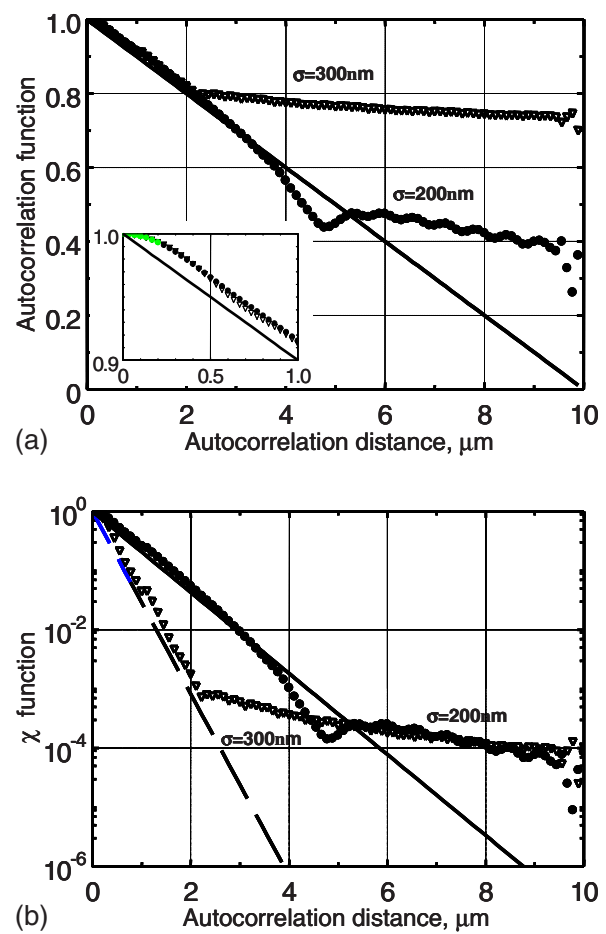

Fig. 3. (Color online) (a) Autocorrelation function versus distance: solid curve, $C=1-\tau / \Lambda$ with $\Lambda=10 \mu \mathrm{m}$; filled circles, reconstructed $C_{\mathrm{IF}}$ for $\sigma=200 \mathrm{~nm}$; inverted triangles, reconstructed $C_{\mathrm{IF}}$ for $\sigma=300 \mathrm{~nm}$. Inset, magnification of the initial behavior. (b) $\chi$ function versus distance for the same case as (a): lines represent the calculated $\chi$ from Eq. (7) for $\sigma=200 \mathrm{~nm}$ (solid line) and $\sigma$ $=300 \mathrm{~nm}$ (dashed line); symbols represent the reconstructed $\chi_{\mathrm{IF}}$ for $\sigma=200 \mathrm{~nm}$ (filled circles) and $\sigma=300 \mathrm{~nm}$ (inverted triangles). 


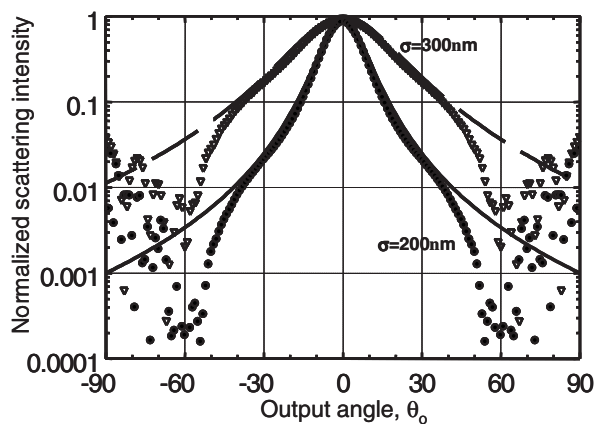

Fig. 4. Normalized scattering intensity versus scattering angle for the same case as in Fig. 3; curves represent the normalized intensity calculated by Eq. (6) with $\sigma=200 \mathrm{~nm}$ (solid), and $\sigma$ $=300 \mathrm{~nm}$ (dashed); symbols represent the reconstructed $I_{\mathrm{IF}}$ for $\sigma=200 \mathrm{~nm}$ (filled circles) and $\sigma=300 \mathrm{~nm}$ (inverted triangles).

tering intensity $I_{\mathrm{IF}}$ calculated from $C_{\mathrm{IF}}$ is compared with the original spectra. Unfortunately, the intensity spectra agree only for small observation angles $\left|\theta_{o}\right|<30^{\circ}$ and fail elsewhere because of the difference between $C$ and $C_{\mathrm{IF}}$.

\section{A. Use of the Singular Value Decomposition}

One way to improve the quality of the reconstruction is to calculate the autocorrelation difference $\Delta C=C-C_{\mathrm{IF}}$ from the intensity difference $\Delta I=I-I_{\mathrm{IF}}$ between the spectra. By this approach, Eq. (5) becomes

$$
I=2 \pi F^{2} \int_{0}^{\infty} J_{o}(2 \pi p \tau) \exp \left[-g\left(1-C_{\mathrm{IF}}-\Delta C\right)\right] \cdot \tau \cdot \mathrm{d} \tau,
$$

where $g=(2 \pi / \lambda)^{2} \sigma^{2}\left(\cos \theta_{i}+\cos \theta_{o}\right)^{2}$. If we assume that the correction $\Delta C$ is small enough that $\exp (g \Delta C) \cong 1+g \Delta C$, Eq. (5) may be linearized into

$$
\Delta I=2 \pi F^{2} \int_{0}^{\infty} J_{o}(2 \pi p \tau) \exp \left[-g\left(1-C_{\mathrm{IF}}\right)\right] \cdot g \cdot \Delta C \cdot \tau \cdot \mathrm{d} \tau
$$

The Fredholm integral in Eq. (11) links $\Delta I\left(\theta_{o}\right)$ to $\Delta C(\tau)$. The relation may be inverted by using singular value decomposition (SVD) [22]. In practice the kernel is decomposed in a set of (orthonormal) singular functions $U_{k}\left(\theta_{o}\right)$ and $V_{k}(\tau)$, which constitute a basis for the direct and inverse spaces [23,24]. In fact the solution $\Delta C$ of the inverse problem may be calculated as a weighted superposition of these singular functions as follows:

$$
\Delta C(\tau)=\sum_{k} a_{k} V_{k}(\tau), \quad \text { where weights } a_{k}=\frac{\left\langle I\left(\theta_{o}\right), U_{k}\left(\theta_{o}\right)\right\rangle}{\mu_{k}}
$$

and where $\mu_{k}$ is the $k$ th singular value and $\langle I U\rangle$ is the integral product in the $\theta_{o}$ space. Keeping in mind the numerical simulations shown in Figs. 3 and 4, it is instructive to follow, in Fig. 5(a), the typical behavior of several singular functions $V_{k}(\tau)$ where $k=1,2,20$, (respectively, curves $1,2,3$ ) and to evaluate the typical values of the weight $a_{k}$, as shown in Fig. $5(\mathrm{~b})$.

It is worth noting that the singular function $V_{k}$ exhibits a number of oscillations increasing with the index $k$,
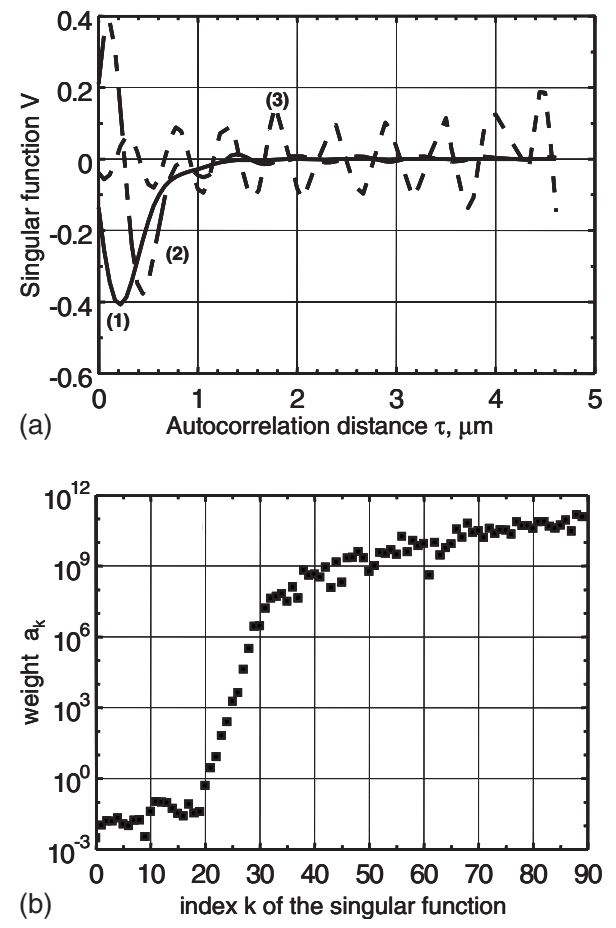

Fig. 5. (a) Singular functions versus distance (micrometers) for the same case as in Fig. 3: curves (1) $k=1$; (2) $k=2$, (3) $k=20$. (b) Weight of the $k$ th singular function versus index $k$ calculated by Eq. (12).

somehow resembling the Fourier series [although these oscillations are aperiodic; see Fig. 5(a)]. Like the case of the Fourier series, the use of high-order singular functions allows us to reconstruct $\Delta C$ with a better spatial resolution. Unfortunately, because of the ill posedness of the inverse problem, the weight $a_{k}$ exhibits a critical amplification for $k>20$, as shown in Fig. 5(b), which introduces unrealistic oscillations in the reconstruction. In practice the summation in Eq. (12) should be always truncated to a maximum index $k_{\mathrm{opt}}$ that represents the optimum maximum number of singular functions used for the reconstruction (i.e., in our case $k_{\text {opt }}=17$ ), which guarantees the requested spatial resolution but without unrealistic oscillations. For example, we report in Fig. 6(a) the result of the improved reconstruction of the linear profile in Figs. 3 with $\sigma=300 \mathrm{~nm}$. The linear profile (curve 1) is reconstructed first by the inverse Fourier transform (open circles) and finally by SVD both using $k_{\mathrm{opt}}=17$ (curve 2) and exceeding that value with $k=20$ (curve 3 ). The correspondent intensity spectra are shown in Fig. 6(b). As expected, when the index $\mathrm{k}$ exceeds $k_{\mathrm{opt}}$, large oscillations appear. The best reconstruction (for $k_{\mathrm{opt}}=17$ ) allows us to extend $\tau_{\max }$ to $2.5 \mu \mathrm{m}$ [see Fig. 6(a)] and to improve substantially the quality of the fit in the whole domain $\left[-90^{\circ}\right.$, $90^{\circ}$ ] as shown in Fig. 6(b).

\section{B. Iterative Procedure}

Looking at Figs. 6, one might be not fully satisfied from the optimum autocorrelation function $C_{1}$ given by SVD with $k_{\text {opt }}$. One reason for the misfit is that the original nonlinear Eq. (5) has been linearized into Eq. (11) only for small $g \cdot \Delta C \ll 1$; the linear inverse problem in Eq. (11) may differ from the nonlinear case in Eq. (5). One way to 


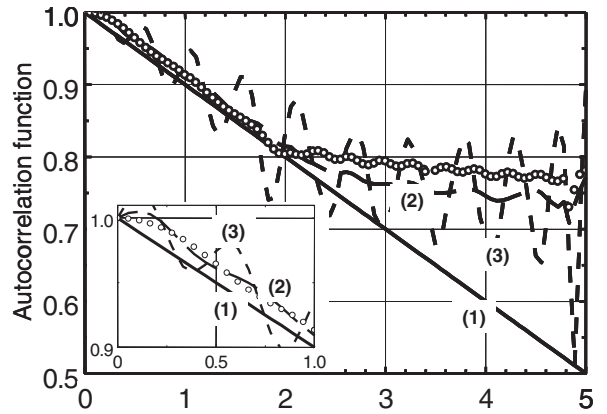

(a)

Autocorrelation distance, $\mu \mathrm{m}$

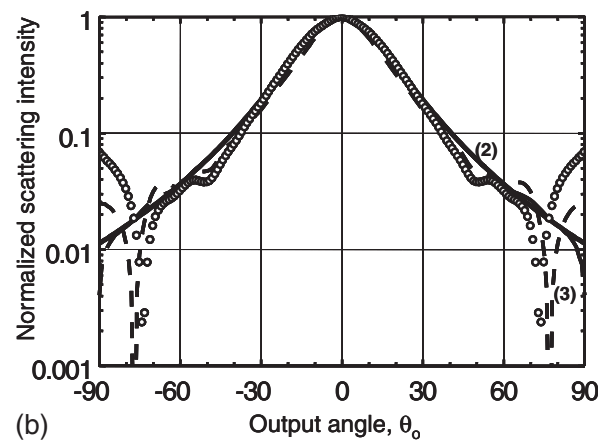

Fig. 6. (a) Reconstructed autocorrelation function versus distance for the case of Fig. 3: (1) linear profile; circles, $C_{\mathrm{IF}}$; (2) $\mathrm{C}$ reconstructed by SVD with $k_{\text {opt }}=17$; (3) $C$ reconstructed by SVD with $k=20$. (b) Normalized scattering intensity versus scattering angle: (1) calculated by Eq. (6); circles, $I_{\mathrm{IF}}$; (2) reconstructed intensity with $k_{\text {opt }}=17$; (3) reconstructed intensity with $k=20$.
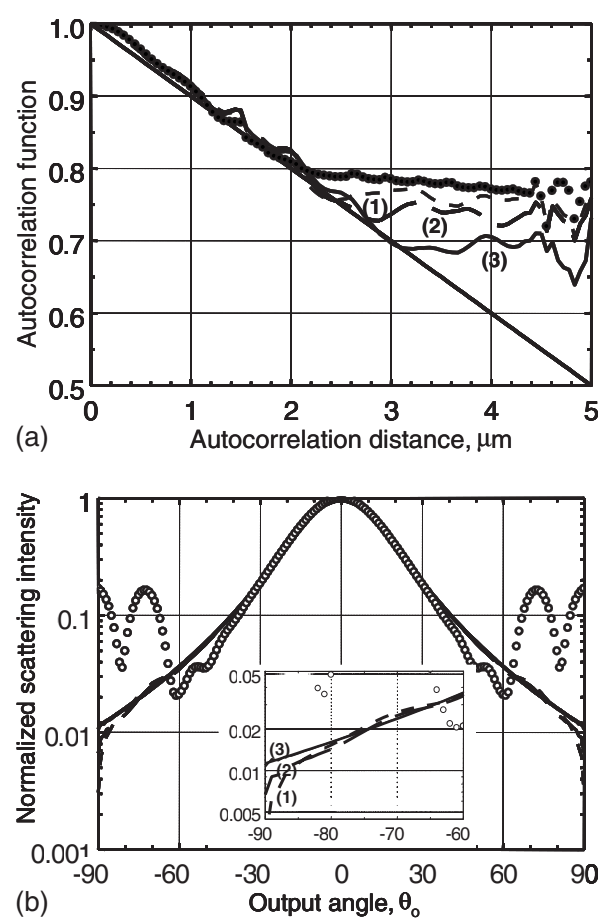

Fig. 7. (a) Reconstructed autocorrelation function versus distance for the case of Fig. 3: solid line, linear profile; circles, $C_{\mathrm{IF}}$; (1) reconstructed $C_{1}$ after the application of one cycle; (2) reconstructed $C_{2}$ after the application of two cycles; (3) reconstructed $C_{3}$ after the application of four cycles. (b) Normalized scattering intensity versus scattering angle: symbols and curves are as in (a). get further improvements is by an iterative procedure; the first solution $C_{1}$ is used again in Eq. (11) as a starting function for the second application of the SVD. The result is an improved solution $C_{2}=C_{1}+\Delta C_{2}$, which diminishes the difference $\Delta I_{2}=I-I_{1}$. This procedure may be iterated $n$ times, by inverting at each step the following integral:

$$
\begin{aligned}
\Delta I_{n}= & I-I_{n-1}=2 \pi F^{2} \int_{0}^{\infty} J_{o}(2 \pi p \tau) \\
& \times \exp \left[-g\left(1-C_{n-1}\right)\right] \cdot g \cdot \Delta C_{n} \cdot \tau \cdot \mathrm{d} \tau .
\end{aligned}
$$

The results of this iterative procedure are shown in Fig. 7, where the improvements in the reconstruction of the autocorrelation function [Fig. 7(a)] and of the intensity spectrum [Fig. 7(b)] are reported for $n=1$ (curve 1), $n=2$ (cuve 2 ), and $n=4$ (curve 3 ). Note that in the case of $C_{4}$ the quantity $\tau_{\max }$ is extended to $3 \mu \mathrm{m}$ Further improvements for $n>4$ are negligible.

\section{EXPERIMENTAL RESULTS}

In this paragraph we report the experimental results performed on a specific rough metallic sample by using several techniques. The description of the investigated sample and the standard measurements of the surface roughness are reported in Subsection 4.A. The experimental setup for the light scattering measurements and the relative experiments are shown in Subsection 4.B. Finally, the reconstruction procedure of the autocorrelation function and the comparison among techniques is discussed in Subsection 4.C.

\section{A. Standard Roughness Measurements}

The investigated sample originally comes from a $1 \mathrm{~mm}$ thick Pd slab subjected to several surface treatments that enhanced the surface roughness. The slab was first rolled and reduced to a $50 \mu \mathrm{m}$ thin foil. Immediately after rolling, the Pd foil was annealed under vacuum conditions for $1 \mathrm{~h}$ at about $900^{\circ} \mathrm{C}$. The high surface roughness was obtained by means of a chemical etching of the sample, which was treated with a solution of nitric and chloridic acid with a 1:3 volume ratio (aqua regia) for about $120 \mathrm{~s}$.

The morphology of the sample has been investigated by atomic force microscopy (AFM). The scans over a typical area of $15 \mu \mathrm{m} \times 15 \mu \mathrm{m}$ of the sample surface are shown in Fig. 8. The estimated rms roughness is here about $\sigma$ $=330 \mathrm{~nm}$, while the autocorrelation length along the $\mathrm{x}$ and $\mathrm{y}$ axes are 1.4 and $1.8 \mu \mathrm{m}$, respectively. However the sample surface is strongly inhomogeneous, and the roughness changes substantially point by point. In order to check the surface roughness at an intermediate scale (millimeter range) a more appropriate measurement has been performed with a stylus by scanning a $3 \mathrm{~mm}$ line as shown in Fig. 8(c).

By a first inspection of Fig. 8(c) the scan passes through zones with different roughness. In particular eleven different zones have been individuated and ordered by capital letters. For each homogeneous zone the roughness, the autocorrelation function (see Fig. 9), and the autocorrelation length have been calculated and are reported in Table 1. Strong variations for both roughness (from 100 to 

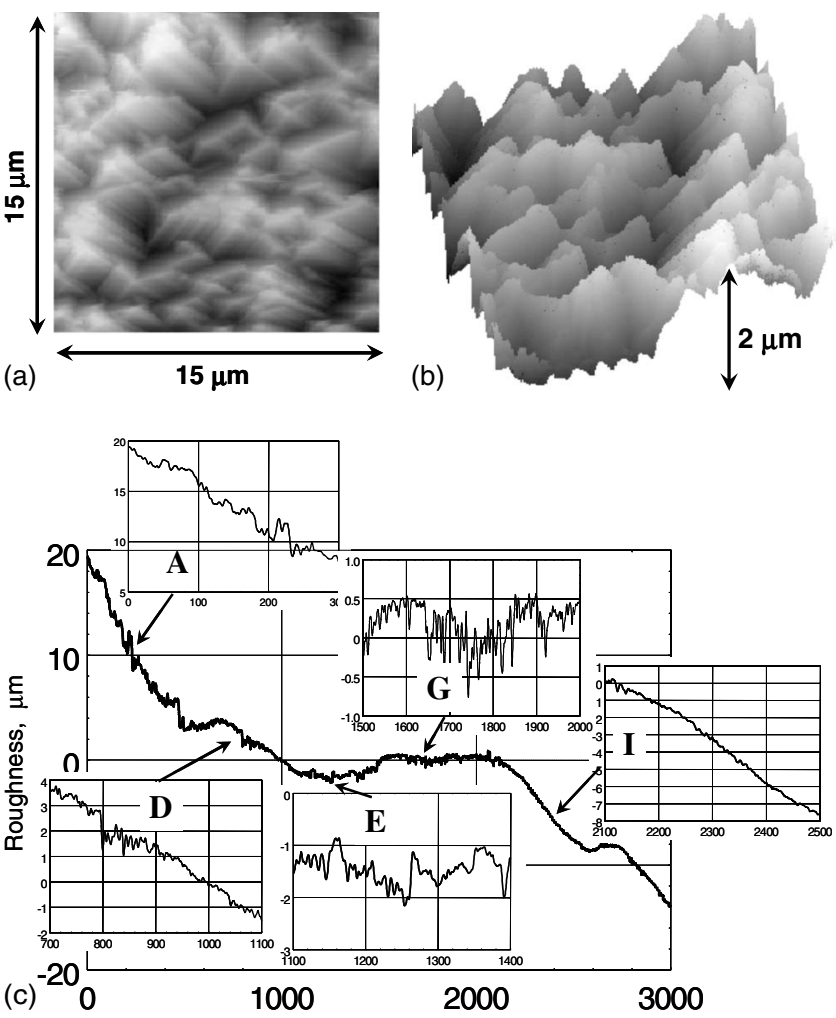

Fig. 8. (a) AFM $x-y$ surface image of the Pd sample. The scanned area is $15 \mu \mathrm{m} \times 15 \mu \mathrm{m}$. (b) Same AFM image in 3D. (c) Stylus scan over a $3 \mathrm{~mm}$ line. The surface roughness (micrometers) is plotted versus the offset (micrometers). Insets, magnifications of five different zones A, D, E, G, I.

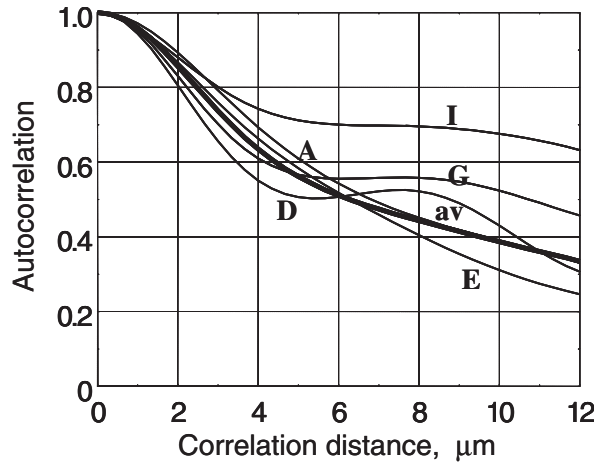

Fig. 9. Normalized autocorrelation function versus autocorrelation distance $\tau$. The autocorrelation $\langle\zeta(x) \cdot \zeta(x+\tau)\rangle$ is calculated from the data shown in Fig. 8(c) in the five zones labeled A, D, E, $\mathrm{G}, \mathrm{I}$; av represents the averaged autocorrelation curve.

$700 \mathrm{~nm}$ ), as well as for the autocorrelation length (from 3 to $11 \mu \mathrm{m}$ ) are clearly evident. It seems worthwhile to average these parameters over all of the $3 \mathrm{~mm}$ line to obtain the quantities $\sigma_{\mathrm{av}}=300 \mathrm{~nm}$ and $\mathrm{ACL}_{\mathrm{av}}=10.5 \mu \mathrm{m}$ (see curve labled "av" in Fig. 9) to be compared with the light scattering measurements as described later in Subsection 4.C.

\section{B. Experimental Setup}

To experimentally measure the scattered light from the rough metallic surface we have designed and realized the in-plane light scattering device shown in Fig. 10 to collect and measure the in-plane scattered intensity even for
Table 1. Results from Stylus Measurements ${ }^{a}$

\begin{tabular}{cccc}
\hline Zone & $\begin{array}{c}\sigma \\
(\mu \mathrm{m})\end{array}$ & $\begin{array}{c}\text { ACL } \\
(\mu \mathrm{m})\end{array}$ & $\begin{array}{c}\text { Scan Length } \\
(\mu \mathrm{m})\end{array}$ \\
\hline A & 0.62 & 10.5 & 300 \\
B & 0.26 & 3 & 100 \\
C & 0.20 & 4.5 & 200 \\
D & 0.26 & 10.5 & 400 \\
E & 0.24 & 8.5 & 300 \\
F & 0.21 & 4.5 & 200 \\
G & 0.25 & - & 500 \\
H & 0.06 & 4 & 100 \\
I & 0.13 & - & 400 \\
L & 0.07 & 3 & 100 \\
M & 0.13 & 6 & 200 \\
Average & 0.30 & 10.5 & 3000 \\
\hline
\end{tabular}

${ }^{\mathrm{a}}$ Zone, zone of the sample; $\sigma, \mathrm{rms}$ roughness; ACL, autocorrelation length. In the last row the average values are calculated.

large scattering output angle $\theta_{o}$ (with respect to the normal to the sample surface) over the whole range $\left[-89^{\circ}\right.$, $\left.+89^{\circ}\right]$. The incident light comes from a $\mathrm{He}-\mathrm{Ne}$ laser at $633 \mathrm{~nm}$. A probe beam of $5 \mathrm{~mW}$ with a diameter of $0.7 \mathrm{~mm}$ is modulated by a mechanical chopper at the frequency $400 \mathrm{~Hz}$ and directed toward the sample surface by a minimirror. The light scattered from the rough sample surface is diffused in all directions. Only the in-plane light scattered in the direction $\theta_{o}$ is filtered by a $1 \mathrm{~mm}$ pinhole and focused by an $f=15 \mathrm{~cm}$ lens over a Si photodiode. The

\section{Angular light scattering measurements. Set-up I}
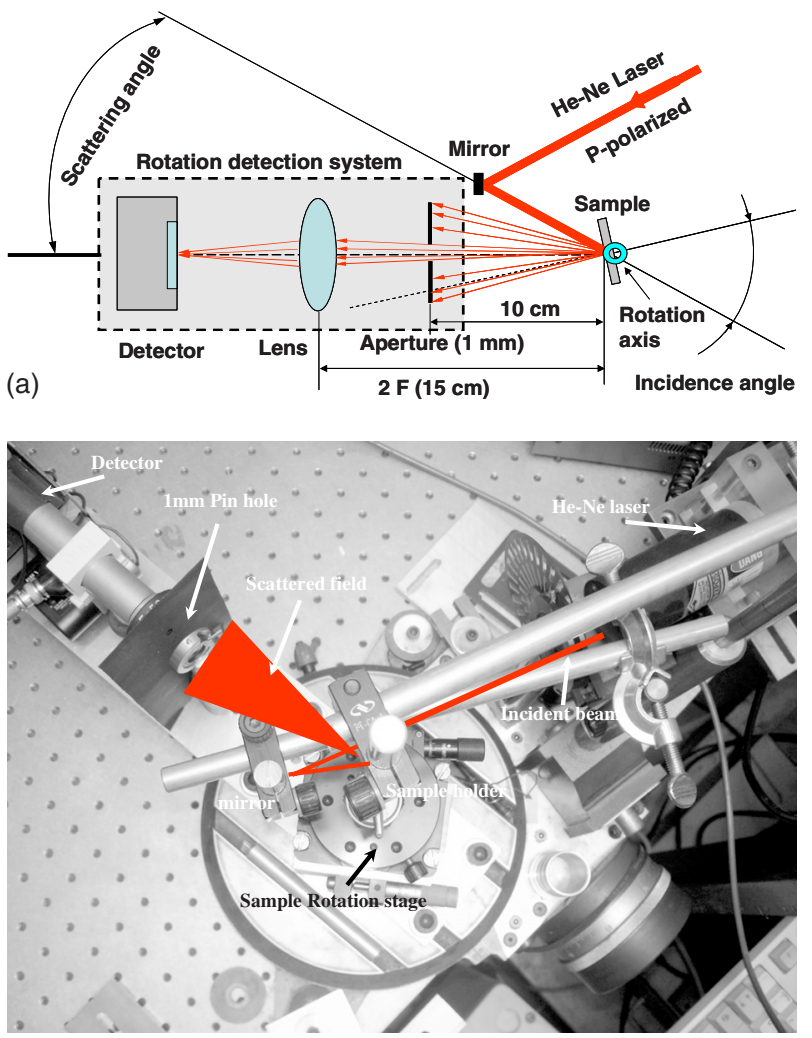

(b)

Fig. 10. (Color online) (a) Schematic setup and (b) experimental setup for the in-plane light scattering measurements. 


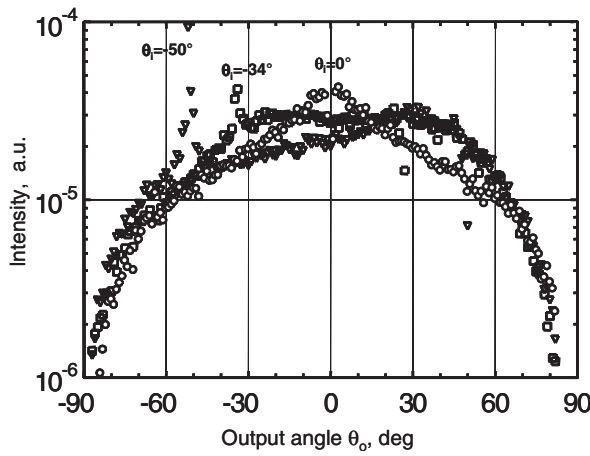

Fig. 11. In-plane scattered light intensity versus scattering angle for $\mathrm{Pd}$ sample. The symbols refer to different incident angles: circles, $\theta_{i}=0^{\circ}$; squares, $\theta_{i}=-34^{\circ}$; inverted triangles, $\theta_{i}=-50^{\circ}$.

signal is finally sent to a lock-in amplifier, which allows the signal to noise ratio to be increased by filtering out signals not syncronous with the mechanical chopper. The angular scans may be performed thanks to two rotation stages: the incident angle $\theta_{i}$ can be adjusted by rotating the sample holder, while the scattering output angle $\theta_{o}$ can be adjusted by rotating the detector arm.

The light scattering measurements have been performed on the rough metallic sample described in Subsection 4.A. The experimental results are reported in Fig. 11. The in-plane scattered intensity has been measured by rotating the detector arm to perform a scan of the scattering angle $\theta_{o}$ in the range $-89^{\circ}$ to $+89^{\circ}$ with an angular step of $1^{\circ}$. During a single set of measurements (scan) the incident angle $\theta_{i}$ has been set to a fixed value: (circles) $\theta_{i}=0^{\circ}$, (squares) $\theta_{i}=-34^{\circ}$, (triangles) $\theta_{i}=-50^{\circ}$. As expected, the maximum of each scan is found in the specular direction $\theta_{\text {out }}=\theta_{i}$. A small backscattering broad peak is also visible for $\theta_{\text {out }} \asymp-\theta_{i}$, even if this phenomenon is not well resolved, because the setup does not allow light to be measured exactly at $\theta_{\text {out }}=-\theta_{i}$ when the incident beam is obscured by the minimirror, which minimizes the dark zone to $1.5^{\circ}$ [see Fig. 10(a)]. In general the shape of all curves is rather flat owing to the high value of the roughness $\sigma \asymp 300 \mathrm{~nm}$ : the dynamic is in this case limited to 2 orders of magnitudes, as is also shown in Figs. 2 and 4 for the theoretical expectation. Moreover, it is worth noting that all curves exhibit a similar behavior for large output angles $\theta_{o}$, which contains information on the initial shape of the autocorrelation function $C(\tau)$.

\section{Reconstruction Procedure and Discussion}

The average reflection of $\mathrm{Pd}$ is around $70 \%$ and is rather flat in a wide range of incidence angles, making the procedure described in Subsection 3.A applicable. However to retrieve the profile $C(\tau)$ only the scattered intensity for normal incidence $\mathrm{I}\left(\theta_{o}, \theta_{i}=0^{\circ}\right.$, circles in Fig. 11) is considered for the inversion procedure, for the following reasons: (a) to prevent the excitation of surface plasmons, which usually may be triggered for larger angles and may lead to a wrong estimate of the material autocorrelation function [25]-a more appropriate model, beyond the aim of the present paper, should be implemented to include these effects; (b) to achieve the maximum signal to noise ratio; (c) to exploit the symmetry of the signal with respect to $\theta_{\text {out }}$.
As a first step Eqs. (8) and (9) are applied to the experimental signal to calculate the inverse Fourier relationship and determine $C_{\mathrm{IF}}(\tau)$ in the range $[0-5 \mu \mathrm{m}]$ as shown in Fig. 12(a) (circles). Some unrealistic oscillations have been averaged as reported in the smoothed curve 1 . The autocorrelation $C_{\text {stylus }}(\tau)$ obtained by stylus (see curve labeled "av" in Fig. 9) is also reported in Fig. 12(a) (solid curve) for comparison. The difference between $C_{\mathrm{IF}}$ and $C_{\text {stylus }}$ is not too surprising in view of the different techniques (contactless optical measurements by a probe beam versus mechanical measurements by a tip in contact with the sample). The main reasons for this difference are summarized as follows: (a) the measurements by stylus are taken with a step of $300 \mathrm{~nm}$ that fixed the maximum spatial resolution. With such accuracy it is impossible to detect surface nanostructures or nanoscale surface patterning. This also explains why $C_{\text {stylus }}$ has a smooth behavior while $C_{\mathrm{IF}}$ has an initial quick drop. (b) In the mechanical measurement by stylus, which is the tip in contact with the sample, some artifacts may appear (induced channels by tip, etc.). (c) The stylus scans the surface roughness along a line, while the optical beam illuminates a large area of the surface. (d) The light scattering measurements are more sensitive to inhomogeneity of the surface, possible surface plasmons in the metal, shadowing effects due to the large roughness, etc. It is therefore somehow improper to require good agreement between the optical and the mechanical measurements. It seems better to require that the data obtained from the
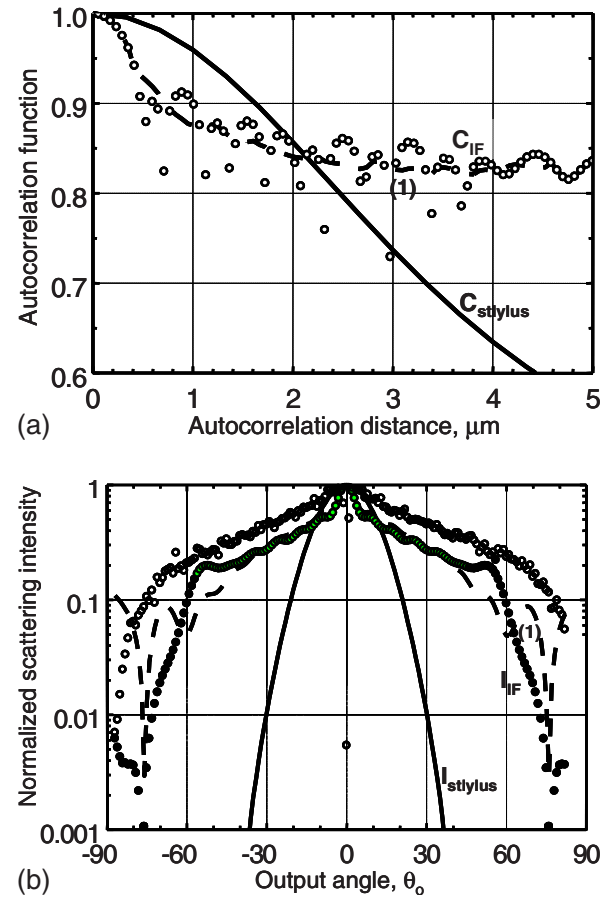

Fig. 12. (Color online) (a) Normalized autocorrelation function versus autocorrelation distance $\tau$ for Pd sample: solid curve, averaged autocorrelation function calculated by stylus as shown in Fig. 9; circles, $C_{\mathrm{IF}}$ autocorrelation calculated by Eq. (9); curve (1) smooting of $C_{\mathrm{IF}}$. (b) In-plane normalized scattered light intensity versus scattering angle (degrees) for $\mathrm{Pd}$ sample and for $\theta_{i}=0^{\circ}$ : open circles, $\mathrm{k}$ experimental results; filled circles, $I_{\mathrm{IF}}$ calculated from $C_{\mathrm{IF}}$ in (a); (1) $I_{\mathrm{IF}}$ calculated by smooting $C_{\mathrm{IF}}$; solid curve, $I_{\text {stylus }}$ calculated from $C_{\text {stylus }}$ in (a). 
scattered measurements are able to retrieve the real intensity distribution.

In order to check the quality of the reconstruction, we compare in Fig. 12(b) the corresponding scattered intensity calculated from Fig. 12(a) by using Eq. (5). It is again evident that $I_{\text {stylus }}$ calculated from $C_{\text {stylus }}$ (solid curve) totally misfits the experimental data (circles), especially at large angles (the surface nanostructures are not seen by this technique). Instead the experimental data are better fitted by $i_{\mathrm{IF}}$ (filled circles) calculated from $C_{\mathrm{IF}}$. A similar quality of the fit is when $C_{\mathrm{IF}}$ is smoothed (curve 1 ). In order to improve the quality of the reconstruction, the quantity $C_{\mathrm{IF}}$ is used as the initial profile for the iterative SVD optimization procedure described in Subsection 3.A.

The results of the procedure are reported in Fig. 13. The profiles $C_{1}$ and $C_{2}$ obtained after the application of one and two cycles of SVD are shown in Fig. 13(a). The iterative procedure practically converges already after two cycles, so that it is irrelevant to show cycles $C_{3}, C_{4}$ etc. The corresponding intensity spectra are reported in Fig. 13(b).

As a conclusion it is worth noting that (a) the quality of the fit becomes excellent with $I_{C 2}$ after already 2 cycles. (b) Further improvements are impossible owing to the level of the experimental noise. (c) The iterative SVD procedure, on the one hand, allows us to find a lower correlation in the range $[2 \mu \mathrm{m}, 5 \mu \mathrm{m}]$, and on the other hand
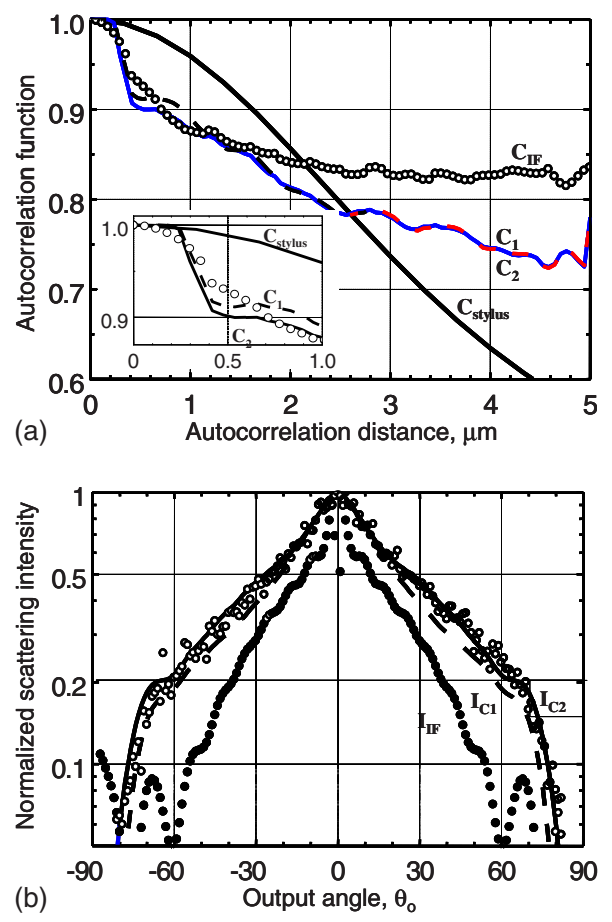

Fig. 13. (Color online) (a) Reconstructed autocorrelation function versus autocorrelation distance $\tau$, for Pd sample: solid curve, averaged autocorrelation function calculated by stylus as shown in Fig. 9; circles, $C_{\mathrm{IF}}$ autocorrelation calculated by Eq. (9); dashed curve, reconstructed $C_{1}$ profile after the application of one SVD cycle; solid curve, reconstructed $C_{2}$ profile after the application of two SVD cycles. (b) In-plane normalized scattered light intensity versus scattering angle (degrees) for Pd sample and for $\theta_{i}=0^{\circ}$ : open circles, experimental results; filled circles, $I_{\mathrm{IF}}$ calculated from $C_{\mathrm{IF}}$; dashed curve, intensity $I_{C 1}$ calculated from $C_{1}$; solid curve, intensity $I_{C 2}$ calculated from $C_{2}$. discovers an initial quick drop of $C(\tau)$ in the range $[0,500 \mathrm{~nm}]$ that is explained by the presence of surface nanostructures, as is clearly visible in the AFM image in Fig. 8(a).

\section{ACKNOWLEDGMENTS}

This work was performed in the framework of a joint collaboration among Dipartimento di Energetica Sapienza Università di Roma, ENEA Frascati, and Energetics LLc. One crucial task of the project was in fact the application of this technique for the nondestructive morphological characterization of some rough metallic samples subjected to an electrolytic process. The authors are indebted with F. Crescentini and M. Magi for technical support.

\section{REFERENCES AND NOTES}

1. Lord Rayleigh, The Theory of Sound (Macmillan, 1896), Vol. II.

2. Lord Rayleigh, "Polish," Nature (London) 64, 385-388 (1901).

3. U. Fano, "The theory of anomalous diffraction gratings and of quasi-stationary waves on metallic surfaces (Sommerfeld's waves)," J. Opt. Soc. Am. 31, 213-222 (1941).

4. S. O. Rice, "Reflection of electromagnetic waves from slightly rough surfaces," Commun. Pure Appl. Math. 4, p. 351-378 (1951).

5. I. M. Fuks, "Theory of radio wave scattering at a rough sea surface," Sov. Radiophys. 9, 513-519 (1966).

6. L. M. Brekhovskikh, "Difrakcya voln na nerovnoj poverhnosti: 1, obschaya teoriya," Zh. Eksp. Teor. Fiz. 23, 275-288 (1952).

7. M. A. Isakovich, "Wave scattering from a statistically rough surface," Zh. Eksp. Teor. Fiz. 23, 305-314 (1952).

8. C. Eckart, "The scattering of sound from the sea surface," J. Acoust. Soc. Am. 25, 66-570 (1953).

9. H. Davies, "The reflection of electromagnetic waves from a rough surface," Proc. Inst. Electr. Eng. 101, 209-214 (1954).

10. P. Beckmann and A. Spizzichino, The Scattering of Electromagnetic Waves from Rough Surfaces (Pergamon, 1963).

11. T. M. Elfouhaily and C. A. Guerin, "A critical survey of approximate scattering wave theories from random rough surfaces," Waves Random Media 14, R1-R40 (2004).

12. J. E. Harvey, A. Krywonos, and C. L. Vernold, "Modified Beckmann-Kirchhoff scattering theory for rough surfaces with large scattering and incident angles," Opt. Eng. 46, 078002 (2007).

13. Y. Sun, "Statistical ray method for deriving reflection models of rough surfaces," J. Opt. Soc. Am. A 24, 724-744 (2007).

14. J.-J. Greffet and M. Nieto-Vesperinas, "Field theory for generalized bidirectional reflectivity: derivation of Helmoltz's reciprocity principle and Kirchhoff's law," J. Opt. Soc. Am. A 15, 2735-2744 (1998).

15. E. Marx, B. Leridon, T. R. Lettieri, J.-F. Song, and T. V. Vorburger, "Autocorrelation functions from optical scattering for one-dimensionally rough surfaces," Appl. Opt. 32, 67-76 (1993).

16. E. L. Church, H. A. Jenkinson, and J. M. Zavada, "Relationship between surface scattering and microtopographic features," Opt. Eng. 18, 125-136 (1979).

17. J. M. Elson and J. M. Bennett, "Vector scattering theory," Opt. Eng. 18, 116-124 (1979). 
18. J. C. Stover, S. A. Serati, and C. H. Gillespie, "Calculation of surface statistics from light scatter," Opt. Eng. 23, 406-412 (1984)

19. J. M. Elson and J. M. Bennett, "Relation between the angular dependence of scattering and the statistical properties of optical surfaces," J. Opt. Soc. Am. 69, 31-47 (1979)

20. I. S. Gradshteyn and I. M. Ryzhik, Table of Integrals, Series and Products (Academic, 1965): $\int_{0}^{\infty} J_{o}(\beta x) \exp (-\alpha x) x \mathrm{~d} x$ $=1 /\left[\alpha^{2}\left(1+(\beta / \alpha)^{2}\right)^{3 / 2}\right]$.

21. The intensity profiles are calculated numerically from Eq. (5), with particular care taken to check the convergence of the integral in the domain $[0, \infty)$. In practice the domain of integration may be truncated to $[0, L=1 \mathrm{~mm}]$ where $L$ is the spot size. The integration of the autocorrelation function $C(\tau)$ is carefully evaluated only in the initial domain $\left[0, \tau_{\max }=10 \mu \mathrm{m}\right]$ with fixed step of $10 \mathrm{~nm}$, while in the final domain $\left[\tau_{\max }, L\right]$, to save computational time, we may reasonably assume $C=0$ during the integration of the $\chi$ function. Nevertheless, in the lucky case of the linear autocorrelation function $C_{b}$, the intensity profile may be expressed in the exact form in Eq. (6). For this reason we use this case as a reference case to check the validity of the inversion procedures.

22. P. C. Hansen, "The truncated SVD as a method for regularization," BIT 27, 534-553 (1987).

23. R. Li Voti, C. Sibilia, and M. Bertolotti, "Photothermal depth profiling by genetic algorithms and thermal wave backscattering," Int. J. Thermophys. 26, 1833-1848 (2005).

24. M. Tomoda, R. Li Voti, O. Matsuda, and O. B. Wright, "Tomographic reconstruction of picosecond acoustic strain propagation," Appl. Phys. Lett. 90, 041114 (2007).

25. K. Seal A. K. Sarychev, H. Noh, D. A. Genov, A. Yamilov, V. M. Shalaev, Z. C. Ying, and H. Cao, "Near-field intensity correlations in semicontinuous metal-dielectric films,"Phys. Rev. Lett. 94, 226101 (2005) 